\title{
As relações de poder e a construção de sentidos sobre o Trabalho Infantil Doméstico (TID): dos media às trabalhadoras
}

\section{Janine Bargas}

\section{Resumo}

Resenha do Livro Comunicação e Trabalho Infantil Doméstico: política, poder, resistências, de Danila Cal, contemplado com o Prêmio Eduardo Peñuela, da Associação de Programas de Pós-Graduação em Comunicação Social (Compós), como a melhor tese de 2015. Publicado em 2016, pela Editora da Universidade Federal da Bahia (EDUFBA). Disponível para Download em https://repositorio.ufba.br/ri/handle/ri/19357

\section{Palavras-Chave}

Comunicação. Trabalho Infantil Doméstico. Poder.

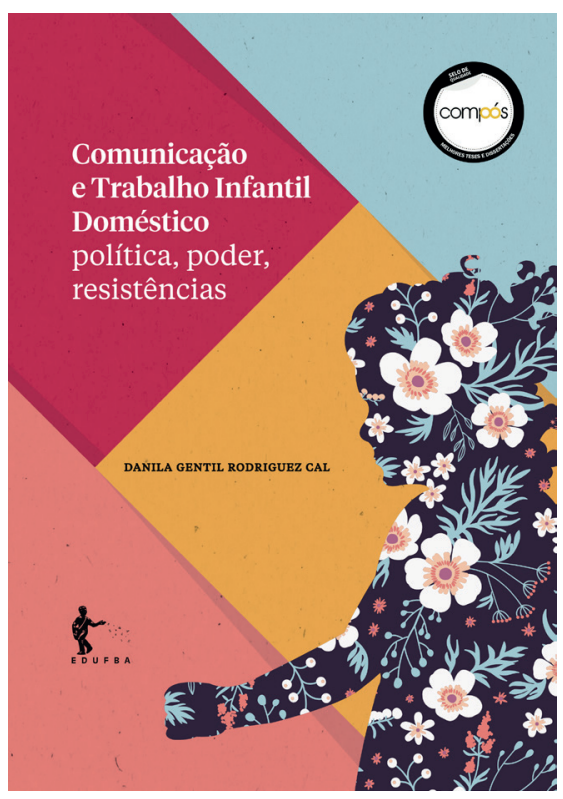

Janine Bargas I ninebargas@gmail.com Doutoranda no Programa de Pós-Graduação em Comunicação Social da Universidade Federal de Minas Gerais - UFMG, Brasil. Pesquisadora dos grupos de pesquisa Mídia e Esfera Pública (EME/ UFMG) e Comunicação, Política e Amazônia (Compoa/UFPA).
A literatura de expressão amazônica está repleta de narrativas que revelam a dura experiência do trabalho infantil doméstico (TID) na região. Prática histórica e culturalmente constituída no Brasil inteiro, o TID encontra no Pará conjuntura favorável à sua reprodução, fundamentada - nos ainda existentes - baixos índices de desenvolvimento humano e econômicos.

Dalcídio Juradir (1960), escritor marajoara, registrou com maestria essas realidades em suas obras, como em Belém do Grão-Pará. Alguns de seus excertos foram usados por Danila Cal para demonstrar, em seu livro Comunicação e Trabalho Infantil Doméstico, o componente sociocultural que envolve esta prática.

Os dados mais recentes sobre 0 TID, publicados em 2013 pelo Fórum Nacional de Prevenção e Erradicação do Trabalho Infantil ${ }^{1}$, com base nos dados da Pesquisa Nacional por Amostra de Domicílios do Instituto Brasileiro de Geografia e Estatística (Pnad/IBGE), de 2008 a 2011, mostram que, proporcionalmente, os estados das regiões Norte e Sul do país apresentam os 
maiores percentuais de crianças e adolescentes trabalhadoras (10,8\% e 10,6\%, respectivamente).

Considerado como um dos trabalhos mais difíceis de serem fiscalizados por ocorrer principalmente nos âmbitos da vida privada, o TID se mostra como um problema sociopolítico. Em seu livro, Danila Cal relata situações de contato com meninas que trabalhavam na casa de seus familiares e amigos, observando seu caráter marcadamente interseccional de gênero e de raça, sendo elas majoritariamente meninas e negras/caboclas.

A autora relata, já na vida adulta, sua experiência de trabalho junto ao Fundo das Nações Unidas para a Infância (Unicef) que a despertou para uma maior atuação junto ao tema. Na sua vida pessoal, viu-se novamente confrontada, pela experiência da maternidade, com a necessidade de alguém que pudesse cuidar do seu filho enquanto trabalhava e, diante disso, não raras vezes foi aconselhada a "acolher uma menina do interior".

\section{0 que Danila Cal apresenta em Comunicação} e Trabalho Infantil Doméstico é resultado de um longo processo de amadurecimento teórico, metodológico e empírico, que iniciou ainda na sua graduação em Comunicação Social, passando pelo mestrado, quando produziu pesquisa acerca do debate público sobre 0 TID nos media e a percepção das mulheres patroas (CAL, 2007). Já no doutorado, junto ao Programa de Pós-Graduação em Comunicação Social da Universidade Federal de Minas Gerais (PPGCOM/ UFMG), a pesquisadora voltou-se às relações de poder subjacentes à prática do trabalho infantil e à construção de sentidos sobre 0 TID pelas próprias meninas e adolescentes trabalhadoras.

A tese, que originou o livro aqui apresentado, editado pela EDUFBA, recebeu o prêmio Eduardo Peñuela, da Associação de Programas de PósGraduação em Comunicação Social (Compós), como a melhor tese de 2015. E não é difícil perceber os méritos do trabalho.

Com linguagem apurada e de leitura fluida, a obra apresenta, pela própria complexidade do tema e de sua abordagem, três seções pré-textuais que respectivamente visam a contextualizar a obra em suas contribuições, feita pela Prof ${ }^{a}$. Dra $^{a}$. Rousiley Maia ${ }^{2}$, um preâmbulo, onde são apresentadas as relações da autora com 0 tema e uma apresentação que introduz 0 caráter mais puramente acadêmico da obra, sintetizando sua abordagem metodológica, principais autores e conceitos, bem como sua estruturação.

Na sequência, seis capítulos corporificam a intenção de Danila Cal de trazer à tona as formas 
de subalternização de meninas paraenses por meio do TID, mas também sua capacidade de resistir e (re)construir sentidos sobre suas realidades. Para isso, a autora reflete sobre 0 TID no seu caráter político, fortemente inspirada em Jürgen Habermas (1984; 1993; 2003; 2009), Jane Mansbridge (1999; 2009) e Axel Honneth ${ }^{3}$ (2003; 2007; 2012), autores para os quais as relações intersubjetivas constituem, de forma dinâmica, 0 político e as gramáticas morais que nos regem em sociedade. A noção de poder, diferentemente de um sem-número de pesquisas das ciências sociais e da comunicação no Brasil, é desenvolvida com inspiração na teórica feminista Amy Allen (1998; 2000; 2013), que a concebe por meio de um tripé analítico onde os sujeitos não são apenas inertes em sua subalternização, mas ativos nas suas formas de suplantação das desigualdades, por meio da resistência e da solidariedade.

0 primeiro capítulo conceitua o TID como uma atividade exercida por pessoas menores de 18 anos em casas de terceiros, e aprofunda os seus aspectos sociais e culturais, enquadrando-o a partir de referências acadêmicas e de organizações sociais, como uma forma de dominação. A autora descreve, então, as condições de vivências de meninas e adolescentes, suas rotinas de trabalho, sua relação com a "família empregadora" e sua família de origem, seus quartos sempre próximos à cozinha e sua vida escolar. Neste momento, as falas de trabalhadoras e patroas são permanentemente evocadas como uma tentativa de se fazer sempre aproximar 0 leitor da realidade sensível vivida por elas.

As ações de advocacy e as campanhas de mobilização e sensibilização realizadas por organismos sociais aparecem no segundo capítulo. Neste momento também são apresentadas ao leitor as normativas legais e as políticas de enfrentamento ao TID, e a experiência de atuação da autora no Programa de Erradicação do Trabalho Infantil (Petid), realizado em Belém do Pará, no ano 2000. Com esta seção, a autora busca discutir a relevância de ações de combate a formas de subordinação que não são necessariamente desenvolvidas por iniciativa das pessoas afetadas, e suas estratégias de comunicação, como campanhas publicitárias e relação com os media.

Pré-político ou político? Para a autora, o TID é político e, para afirmá-lo como tal, recorre a uma revisão de literatura onde autoras como Arendt (1991; 1994), Mansbridge (1999) e Oskin (2000) e autores como David Held (1987) e Habermas (2003) são evocados no que dizem sobre a conexão imanente entre a vida cotidiana e as ações de Estado que, por excelência, toca o político. Na terceira grande seção da obra, percebemos, então, mais sensivelmente a concepção do político atribuída ao TID, que se faz necessária pelo seu 
caráter quase "invisível" na perspectiva do social e do público, e a abordagem que Danila Cal faz sobre as relações de poder subjacentes ao TID.

É neste momento também que a autora nos deixa clara uma das inovações de seu trabalho de pesquisa quando foge de uma perspectiva dicotômica do poder, onde, de um lado, está o opressor e, do outro, o oprimido. Amy Allen (2000) oferece aqui uma perspectiva alternativa a essas formas dicotômicas de pesar as relações de poder - como é próprio das epistemologias feministas - ao apresentar o power over, o power to, e 0 power with, podendo ser traduzidos, respectiva e literalmente como "poder sobre", "poder para" e "poder com".

Em termos analíticos, a costura que Cal faz com Allen, também com a ajuda de autores clássicos, como Max Weber e Pierre Bourdieu, quer nos dizer sobre essas três dimensões do poder: a) 0 exercício da dominação sobre 0 outro a fim de obter um resultado desejado, que se sustenta por meio de práticas costumeiras e até institucionalizadas; b) a construção de resistência e 0 empoderamento por meio da perseguição de projetos de vida, capturas de possibilidades de consecução de objetivos; e c) construção de vontades comuns por meio de solidariedades, a partir de laços de identificação, especialmente. Essas dimensões, para Cal, possuem "diferentes repercussões para a configuração política do TID" (CAL, 2016, p. 103).
Os contextos comunicacionais acerca do tema e o percurso metodológico da pesquisa estão no quarto capítulo. No primeiro momento desta seção, Danila Cal reforça a importância das ações de comunicação no combate ao TID por sua capacidade de publicação da questão, além de ampliação do debate público acerca do tema. Foram analisadas 136 matérias, veiculadas entre 2000 e 2009, nos principais jornais impressos do Pará (0 Liberal e Diário do Pará), com uso de técnicas quantitativas e qualitativas. Aqui, a autora menciona também sua experiência de pesquisa no mestrado, que aponta uma distinção entre 0 discurso de combate ao TID e a percepção das empregadoras: se, por um lado, houve um amplo discurso de advocacy nos meios de comunicação, por outro, as famílias não se viam em uma situação de exploração de meninas e adolescentes. Faltara, então, ouvir as perspectivas das trabalhadoras.

Grupos focais foram considerados os melhores instrumentos para que a pesquisadora pudesse captar de forma nuançada, num primeiro e num segundo plano, a percepção do poder e a construção de sentido das meninas, adolescentes e ex-trabalhadoras domésticas sobre 0 TID. Isso porque os grupos permitem mais do que questionamentos de um pesquisador a um entrevistado, mas sim um compartilhamento de experiências entre as participantes.

Ao todo, a autora realizou sete grupos focais com meninas e mulheres com perfis distintos. Neste segundo momento da seção, Danila Cal faz uma 
descrição minunciosa dos caminhos, das questões e da logística de realização dos grupos focais, abordando, também, o momento exploratório, as dificuldades operacionais e éticas que envolvia sua relação com as interlocutoras da pesquisa. Esta é outra grande contribuição da obra que se concentra no caráter metodológico e ético que envolve pesquisa sobre temas sensíveis.

0 quinto capítulo apresenta o uso das categorias analíticas e do corpus descritos nas seções anteriores. São realizados aqui o que a autora chama de "movimentos analíticos" (CAL, 2016, p. 150): da observação sistemática do material dos media e das falas das trabalhadoras à análise das reverberações políticas do TID a partir dos sujeitos envolvidos. Entre os principais pontos da análise, Danila Cal destaca as relações temáticas entre o TID e a "violência", como forma mais óbvia de dominação, e a "educação", como a área da vida das trabalhadoras mais afetada pela prática. Por meio de tabelas, a autora relaciona suas categorias aos achados da pesquisa empírica, facilitando ao leitor a compreensão de sua tessitura teórico-metodológica.

Para a autora, a configuração política acerca do TID nos media apontam para a ideia de power over (Allen, 2000), isto é, do constrangimento de sujeitos oprimidos, como uma dominação internalizada e naturalizada pelas meninas trabalhadoras. A exceção estava nas meninas atendidas pelo Petid que apareciam como fontes em reportagens, onde apresentavam opiniões mais críticas sobre 0 TID.
Na perspectiva das trabalhadoras afetadas, a configuração política foi mais complexa e ambígua: todas as dimensões do poder usadas como categorias analíticas se relacionavam de modo a construir entendimentos distintos acerca do TID. Neste aspecto, as trabalhadoras referiam-se particularmente à relação com a "família empregadora", a ideia de que se trata de um trabalho e não de uma ajuda, ao insucesso escolar como algo quase que natural pelas condições apresentadas, entre outros pontos.

A despeito disso, possibilidades de resistência foram postas especialmente pelas extrabalhadoras sindicalizadas, como a necessidade de lutas individuais pela melhoria das condições de vida. A solidariedade apareceu, principalmente, quando o componente de gênero era tratado entre as trabalhadoras como uma violência que as fadava a determinadas atividades e condições.

Generosamente, portanto, Danila Cal, de forma nítida e bem encadeada, apresenta 0 saber fazer da pesquisa, bem como o entrelaçamento teórico bem definido, sempre com revisões críticas da literatura. Para cada seção do livro, a autora se preocupou em convidar o leitor ao texto; de forma sensível, aproximou-nos da história de cada trabalhadora doméstica transformando o que poderia ser um austero resultado de pesquisa acadêmica, numa sensibilização permanente para as vidas e para 0 tema. 
A publicização das realidades vividas por muitas meninas, adolescentes e mulheres trabalhadoras domésticas, assim como a menção enfática às contribuições de autoras feministas - além de Allen, são citadas Flávia Biroli, Gayatri Spivak e Linda Alcoff, por exemplo - própria de uma pesquisa epistemologicamente feminista, revela ainda seu compromisso com as interlocutoras e com a transformação social das desigualdades.

E, ao apontar novos horizontes de investigação sobre 0 trabalho infantil doméstico, sobre as escolhas e o percurso metodológico, Danila Cal cumpre também a função de instigar pesquisas de fôlego e qualidade que podem evocar referências da filosofia política, da sociologia, da antropologia e do feminismo em uma pesquisa eminentemente comunicacional.

\section{Referências}

ALLEN, Amy. Rethinking Power. Hypatia, [S.l], v. 13, n. 1, p. 21-40, 1998. Disponível em < https://www. jstor.org/stable/3810605?seq=1\#page_scan_tab_ contents > . Acesso em: 2 jan. 2017.

The power of feminist theory: domination, resistance, solidarity. Boulder: Westview Press, 2000. Feminist Perspective on Power. The Stanford Ecyclopedia os Philosophy. USA: Edward N. Zalta (Ed.), 2013. Disponível em: < https://plato.stanford. edu/entries/feminist-power/> . Acesso em: 2 jan. 2017.

ARENDT, Hannah. A condição Humana [1958]. Rio de Janeiro: Forense Universitária, 1991.

Sobre a violência. Rio de Janeiro: Relume Dumará, 1994.
CAL, Danila. Entre o privado e o público: contextos comunicativos, deliberação e trabalho infantil doméstico. 2007. 152f. Dissertação (Mestrado em Comunicação Social). Faculdade de Filosofia e Ciências Humanas, Universidade Federal de Minas Gerais, Belo Horizonte, 2007.

\section{Comunicação e Trabalho Infantil}

Doméstico. Política, poder, resistências. - Salvador: EDUFBA, 2016.

HABERMAS, Jürgen. The theory of communicative action: rason and the rationalization of society. Volume I. Tradução: Thomas McCarthy. Boston: Beacon Press, 1984.

0 conceito de poder em Hannah Arendt. In: FREITAG, B.; ROUANET, S. P. (Orgs.) Habermas Sociologia. São Paulo: Ática, 1993, p. 89-108.

. 0 papel da sociedade civil e da esfera pública política. In: HABERMAS, J. Direito e Democracia: entre a facticidade e validade. Volume II. Rio de Janeiro: Tempo Brasileiro, 2003.

Europe: the faltering Project. Cambridge: Polity Press, 2009.

HELD, David. 0 que a democracia deveria significar hoje? In: HELD, D. Modelos de Democracia. Belo Horizonte: Paidéia, 1987.

HONNETH, Axel. Luta por reconhecimento: a gramática moral dos conflitos sociais. São Paulo: Ed 34, 2003.

Recognition as ideology: the connection between morality and power. In: HONNETH, A. The I in WE: studies in the theory of recognition. Cambridge: Polity Press, 2007. Recognition as Ideology. In: VAN DEN BRINK, B; OWEN, D. (Ed.) Recognition and power: Axel Honneth and the tradition of critical social theory. New York: Cambridge University Press, 2007. P. 323-347.

JURANDIR, Dalcídio. Belém do Grão-Pará. São Paulo: Livraria Martins Editora, 1960. 
MANSBRIDGE, Jane. Everyday talk in Deliberative System. In: MACEDO, S. (ed.). Deliberative Politics: essays on democracy and disagreement. 0xford: 0xford University Press, 1999. p. 211-239.

A conversação cotidiana no Sistema

deliberative. Tradução: Ângela Cristina Salgueiro Marques. In: MARQUES, A. C. S. (Org.) A deliberação pública e suas dimensões sociais, políticas e comunicativas: textos fundamentais. Belo Horizonte: Autêntica Editora, 2009. p. 207-238. 


\begin{tabular}{|c|c|}
\hline $\begin{array}{l}\text { Power relations and the } \\
\text { construction of meanings about } \\
\text { Domestic Child Work: From the } \\
\text { media to the workers }\end{array}$ & $\begin{array}{l}\text { Las relaciones de poder y la } \\
\text { construcción de sentido en el trabajo } \\
\text { infantil doméstico (TID): los medios } \\
\text { de comunicación a los trabajadores }\end{array}$ \\
\hline $\begin{array}{l}\text { Abstract } \\
\text { Book Review Communication and Child Domestic } \\
\text { Work: politics, power, resistances, by Danila Cal, } \\
\text { awarded with the Eduardo Peñuela Prize, by the } \\
\text { Association of Postgraduate Programs in Social } \\
\text { Communication (Compós), as the best thesis of } 2015 . \\
\text { Published in 2016, by the Federal University of Bahia } \\
\text { (EDUFBA). Available for Download at < https:// } \\
\text { repositorio.ufba.br/ri/handle/ri/19357>. } \\
\text { Keywords } \\
\text { Communication. Domestic Child Work. Power. }\end{array}$ & $\begin{array}{l}\text { Resumen } \\
\text { Revisión del Libro de comunicación y el trabajo infantil } \\
\text { doméstico: la política, el poder, la resistencia, Danila } \\
\text { Cal, galardonado con el Premio Eduardo Peñuela, el } \\
\text { Programa de Posgrado en Asociación de Comunicación } \\
\text { Social (Compós) como la mejor tesis de } 2015 \text { Publicado } \\
2016 \text { por la Editora Universidad Federal de Bahía } \\
\text { (EDUFBA). Disponible para su descarga em< https:// } \\
\text { repositorio.ufba.br/ri/handle/ri/19357 >. } \\
\text { Palabras clave } \\
\text { Comunicación. Trabajo Infantil Doméstico. Poder. }\end{array}$ \\
\hline
\end{tabular}




\section{Expediente}

A revista E-Compós é a publicação científica em formato eletrônico da Associação Nacional dos Programas de Pós-Graduação em Comunicação (Compós). Lançada em 2004, tem como principal finalidade difundir a produção acadêmica de pesquisadores da área de Comunicação, inseridos em instituições do Brasil e do exterior.

\section{E-COMPÓS I www.e-compos.org.br I E-ISSN 1808-2599}

Revista da Associação Nacional dos Programas de Pós-Graduação em Comunicação. Brasília, v.20, n.2, maio/ago. 2017. A identificação das edições, a partir de 2008, passa a ser volume anual com três números. Indexada por Latindex I www.latindex.unam.mx

\section{CONSELHO EDITORIAL}

Alda Cristina Silva da Costa, Universidade Federal do Pará, Brasil Alfredo Luiz Paes de Oliveira Suppia, Universidade Estadual de Campinas, Brasil Álvaro Larangeira, Universidade Tuiuti do Paraná, Brasil Ana Carolina D. Escosteguy, Pontifícia Universidade Católica do Rio Grande do Sul, Brasil Ana Regina Barros Rego Leal, Universidade Federal do Piauí, Brasil Ana Carolina Rocha Pessôa Temer, Universidade Federal de Goiás, Brasil Andrea França, Pontifícia Universidade Católica do Rio de Janeiro, Brasil André Luiz Martins Lemos, Universidade Federal da Bahia, Brasil Angela Cristina Salgueiro Marques, Faculdade Cásper Líbero, Brasil Ângela Freire Prysthon, Universidade Federal de Pernambuco, Brasil Antonio Carlos Hohlfeldt, Pontifícia Universidade Católica do Rio Grande do Sul, Brasil Arthur Ituassu, Pontifícia Universidade Católica do Rio de Janeiro, Brasil Bruno Campanella, Universidade Federal Fluminense, Brasil Cláudio Novaes Pinto Coelho, Faculdade Cásper Líbero, Brasil Carlos Eduardo Franciscato, Universidade Federal de Sergipe, Brasil Denise Tavares da Silva, Universidade Federal Fluminense, Brasil Eduardo Vicente, Universidade de São Paulo, Brasil Eliza Bachega Casadei, Escola Superior de Propaganda e Marketing - SP, Brasil Elizabeth Nicolau Saad Corrêa, Universidade de São Paulo, Brasil Erick Felinto de Oliveira, Universidade do Estado do Rio de Janeiro, Brasil Erly Vieira Júnior, Universidade Federal do Espírito Santo, Brasil Francisco de Assis, FIAM-FAAM Centro Universitário, Brasil Francisco Elinaldo Teixeira, Universidade Estadual de Campinas, Brasil Frederico de Mello Brandão Tavares, Universidade Federal de Ouro Preto, Brasil Gabriela Reinaldo, Universidade Federal do Ceará, Brasil

Gilson Vieira Monteiro, Universidade Federal do Amazonas, Brasil Gustavo Daudt Fischer, Universidade do Vale do Rio dos Sinos, Brasi Itania Maria Mota Gomes, Universidade Federal da Bahia, Brasil Jiani Adriana Bonin, Universidade do Vale do Rio dos Sinos, Brasil José Afonso da Silva Junior, Universidade Federal de Pernambuco, Brasil José Luiz Aidar Prado, Pontifícia Universidade Católica de São Paulo, Brasi
Juçara Gorski Brittes, Universidade Federal de Ouro Preto, Brasil Juliana Freire Gutmann, Universidade Federal da Bahia, Brasil Laura Loguercio Cánepa, Universidade Anhembi Morumbi, Brasil Letícia Cantarela Matheus, Universidade do Estado do Rio de Janeiro, Brasil Liziane Soares Guazina, Universidade de Brasília, Brasil Luíza Mônica Assis da Silva, Universidade Católica de Brasília, Brasil Maria Ataide Malcher, Universidade Federal do Pará, Brasil Maria Elisabete Antonioli, Escola Superior de Propaganda e Marketing - SP, Brasil Maria das Graças Pinto Coelho, Universidade Federal do Rio Grande do Norte, Brasil Marcel Vieira Barreto Silva, Universidade Federal da Paraiba, Brasil Marcia Tondato, Escola Superior de Propaganda e Marketing, Brasil Marli Santos, Universidade Metodista de São Paulo, Brasil Márcio Souza Gonçalves, Universidade do Estado do Rio de Janeiro, Brasil Mauricio Mario Monteiro, Universidade Anhembi Morumbi, Brasil Mauricio Ribeiro da Silva, Universidade Paulista, Brasil Mauro de Souza Ventura, Universidade Estadual Paulista, Brasil Mayka Castellano, Universidade Federal Fluminense, Brasi Micael Maiolino Herschmann, Universidade Federal do Rio de Janeiro, Brasil Mozahir Salomão Bruck, Pontifícia Universidade Católica de Minas Gerais, Brasil Nísia Martins Rosario, Universidade Federal do Rio Grande do Sul, Brasil Potiguara Mendes Silveira Jr, Universidade Federal de Juiz de Fora, Brasil Rafael Grohmann, FIAM-FAAM - Centro Universitário, Brasil Raquel Ritter Longhi, Universidade Federal de Santa Catarina, Brasil Regiane Regina Ribeiro, Universidade Federal do Paraná, Brasil Roberto Elísio dos Santos, Universidade Municipal de São Caetano do Sul, Brasil Rodolfo Rorato Londero, Universidade Estadual de Londrina, Brasil Sérgio Luiz Gadini, Universidade Estadual de Ponta Grossa, Brasil Simone Maria Andrade Pereira de Sá, Universidade Federal Fluminense, Brasil Simone Maria Rocha, Universidade Federal de Minas Gerais, Brasil Suzana Reck Miranda, Universidade Federal de São Carlos, Brasil Tarcyanie Cajueiro Santos, Universidade de Sorocaba, Brasil Tatiana Oliveira Siciliano, Pontifícia Universidade Católica do Rio de Janeiro, Brasil Veneza Mayora Ronsini, Universidade Federal de Santa Maria, Brasil

\section{CONSELHO CIENTÍFICO}

Cristiane Freitas Gutfreind, Pontifícia Universidade Católica do Rio Grande do Sul, Brasil | Eduardo Antônio de Jesus, Universidade Federal de Minhas Gerais, Brasil | Eduardo Morettin, Universidade de São Paulo, Brasil I Irene de Araújo Machado, Universidade de São Paulo, Brasil I Miriam de Souza Rossini, Universidade Federal do Rio Grande do Sul, Brasil

\section{COMISSÃO EDITORIAL}

Eduardo Antonio de Jesus, Universidade Federal de Minas Gerais, Brasil I Igor Pinto Sacramento, Universidade Federal do Rio de Janeiro, Brasil I Kelly Cristina de Souza Prudencio, Universidade Federal do Paraná, Brasil | Osmar Gonçalves dos Reis Filho, Universidade Federal do Ceará, Brasil

\section{CONSULTORES AD HOC}

Cesar Augusto Baio Santos, Universidade Federal do Ceará, Brasil I Lilian França Universidade Federal de Sergipe, Brasil I Maria Aparecida Baccega, Escola Superior de Propaganda e Marketing, Brasil I Márcia Benetti, Universidade Federal do Rio Grande do Sul, Brasil I Miguel Serpa Pereira, Pontifícia Universidade Católica do Rio de Janeiro, Brasil | Renato Essenfelder, Escola Superior de Propaganda e Marketing, Brasil

\section{EQUIPE TÉCNICA}

ASSISTENTE EDITORIAL Márcio Zanetti Negrini | REVISÃO DE TEXTOS Melina Santos | EDITORAÇÃO ELETRÔNICA Roka Estúdio I IMAGEM DE CAPA Silas de Paula

COMPÓS I www.compos.org.br

Associação Nacional dos Programas de Pós-Graduação em Comunicação

Presidente

Marco Roxo

Programa de Pós-Graduação em Comunicação - UFF marcos-roxo@uol.com.br

Vice-Presidente Isaltina Gomes Programa de Pós-Graduação em Comunicação - UFPE isaltina@gmail.com

Secretária-Geral

Gisela Castro

Programa de Pós-Graduação em Comunicação e Práticas de Consumo - ESPM castro.gisela@gmail.com

CONTATO I revistaecompos@gmail.com 